\title{
Editorial
}

\section{Mobile Cyber-Physical System}

\author{
Xiping Hu, ${ }^{1}$ Jun Cheng, ${ }^{1}$ Xitong Li, ${ }^{2}$ Wei Tan, ${ }^{3}$ Qiang Liu, ${ }^{4}$ and Zhengguo Sheng ${ }^{5}$ \\ ${ }^{1}$ Shenzhen Institutes of Advanced Technology, Chinese Academy of Sciences, Shenzhen, China \\ ${ }^{2}$ HEC Paris, Paris, France \\ ${ }^{3}$ IBM Thomas J. Watson Research Center, Yorktown Heights, NY, USA \\ ${ }^{4}$ National University of Defense Technology, Changsha, China \\ ${ }^{5}$ University of Sussex, Brighton, UK \\ Correspondence should be addressed to Xiping Hu; xipingh@bravolol.com
}

Received 15 May 2017; Accepted 16 May 2017; Published 3 August 2017

Copyright (C) 2017 Xiping Hu et al. This is an open access article distributed under the Creative Commons Attribution License, which permits unrestricted use, distribution, and reproduction in any medium, provided the original work is properly cited.

In recent years, the capabilities of contemporary mobile devices have been improving a lot. These capabilities, such as significant computational resources, multiple communication radios, various sensor modules, and high level programming languages, enable mobile devices to form mobile cyber-physical system (CPS) in our daily lives. Mobile CPS integrates distributed sensing with computing and ubiquitous connectivity of Internet. Also, mobile CPS coordinates computational, virtual, and physical resources and facilitates the interaction of digital world with physical world, potentially driving the pervasive effect in the citizens' everyday life anytime and anywhere. Thus, mobile CPS could provide a convenient and economical platform that facilitates sophisticated and ubiquitous intelligent applications between humans and the surrounding physical world. Mobile CPS could find applicability in multiple areas and disciplines, including (but not limited to) (1) mobile intelligent robots and robotics systems, making use of multiple smart sensors, mobile devices, intelligent service, cloud robots, and so on, to improve efficiency and scalability in processing of complex tasks that are impossible under local resource constraints in different application areas; (2) intelligent transportation systems, for example, leveraging sensing, computing, and communication capabilities with control vehicles in the physical world to deal with the challenges of safe (e.g., decreasing time latency of reaction in traffic accidents), efficient, and green transportations; and (3) smart living technologies, for example, smart city, environmental monitoring, healthcare systems, and smart grid, which improve intelligence, convenience, operational safety, and green energy of human society. The purpose of this special issue aims to involve multidiscipline research contributions of unpublished research on the recent methodologies, innovations, and experimental validations regarding mobile CPS, so as to facilitate the real-world deployment of such systems in the future. In this special issue on mobile CPS, we have invited a few papers that achieve such goal.

The paper "Sliding Window Based Feature Extraction and Traffic Clustering for Green Mobile Cyber-Physical Systems" focuses on a green mobile cyber-physical system to ensure network coverage and to reduce the total energy consumption, which proposes a feature extraction method using sliding window to extract the distribution feature of mobile user equipment (UE). Furthermore, traffic clustering analysis and corresponding optimized control strategy are presented for the rapid control of base stations. Experimental results demonstrate the superior performance of the proposed method in terms of UE coverage compared with the grid method.

The paper "An Anonymous Access Authentication Scheme Based on Proxy Ring Signature for CPS-WMNs" proposes a novel anonymous access authentication scheme based on proxy ring signature to address the anonymous access authentication issue for CPS-Wireless Mesh Network (CPS-WMN). In the scheme, a hierarchical authentication architecture is presented firstly. Then, intergroup and intragroup anonymous mutual authentication are carried out through proxy ring signature mechanism and certificateless signature mechanism, respectively. A formal security proof with SVO logic and simulation results demonstrate the security and the performance of the proposed scheme. 
The paper "Improved Object Proposals with Geometrical Features for Autonomous Driving" aims at generating high quality object proposals for object detection in autonomous driving, which proposes several geometrical features suited for autonomous driving and integrates them into current general proposal generation methods. Experiments over KITTI benchmark demonstrate the significant performance improvement of the existing methods by using the proposed geometrical features.

The paper "A Formal Approach to Verify Parameterized Protocols in Mobile Cyber-Physical Systems" targets the challenging task of protocol verification in mobile CPS and proposes a formal approach to verify the safety properties of parameterized protocols. In this approach, the protocol is modeled as a Petri net using counterabstraction; then a verification algorithm is presented to verify the Petri net model. Experimental results show higher capability but lower memory consumption compared with existing approaches.

The paper "Design and Voluntary Motion Intention Estimation of a Novel Wearable Full-Body Flexible Exoskeleton Robot" proposes to use steel wire as the flexible transmission medium and design a group of special wire locking structures. Moreover, passive points for partial joints of the exoskeleton are designed and a novel gait phase recognition method for full-body exoskeletons is proposed. Experimental results demonstrate the performance of the proposed method in terms of high correct rates of motion pattern classification and phase recognition.

The paper "Efficient Physical Layer Secret Key Generation and Authentication Schemes Based on Wireless ChannelPhase" focuses on the inefficiency of current physical-layer secret key generation schemes and the unpractical assumption of the pre-known knowledge of the shared key in existing authentication schemes. Then, a novel physical-layer secret key generation scheme and an authentication scheme for Orthogonal Frequency-Division Multiplexing (OFDM) systems are proposed, which both exploit the randomness and reciprocity of the channel-phase response. Simulation results demonstrate the superior performance of the proposed schemes under the NIST randomness test and various attacks.

\author{
Xiping $\mathrm{Hu}$ \\ Jun Cheng \\ Xitong $\mathrm{Li}$ \\ Wei Tan \\ Qiang Liu \\ Zhengguo Sheng
}



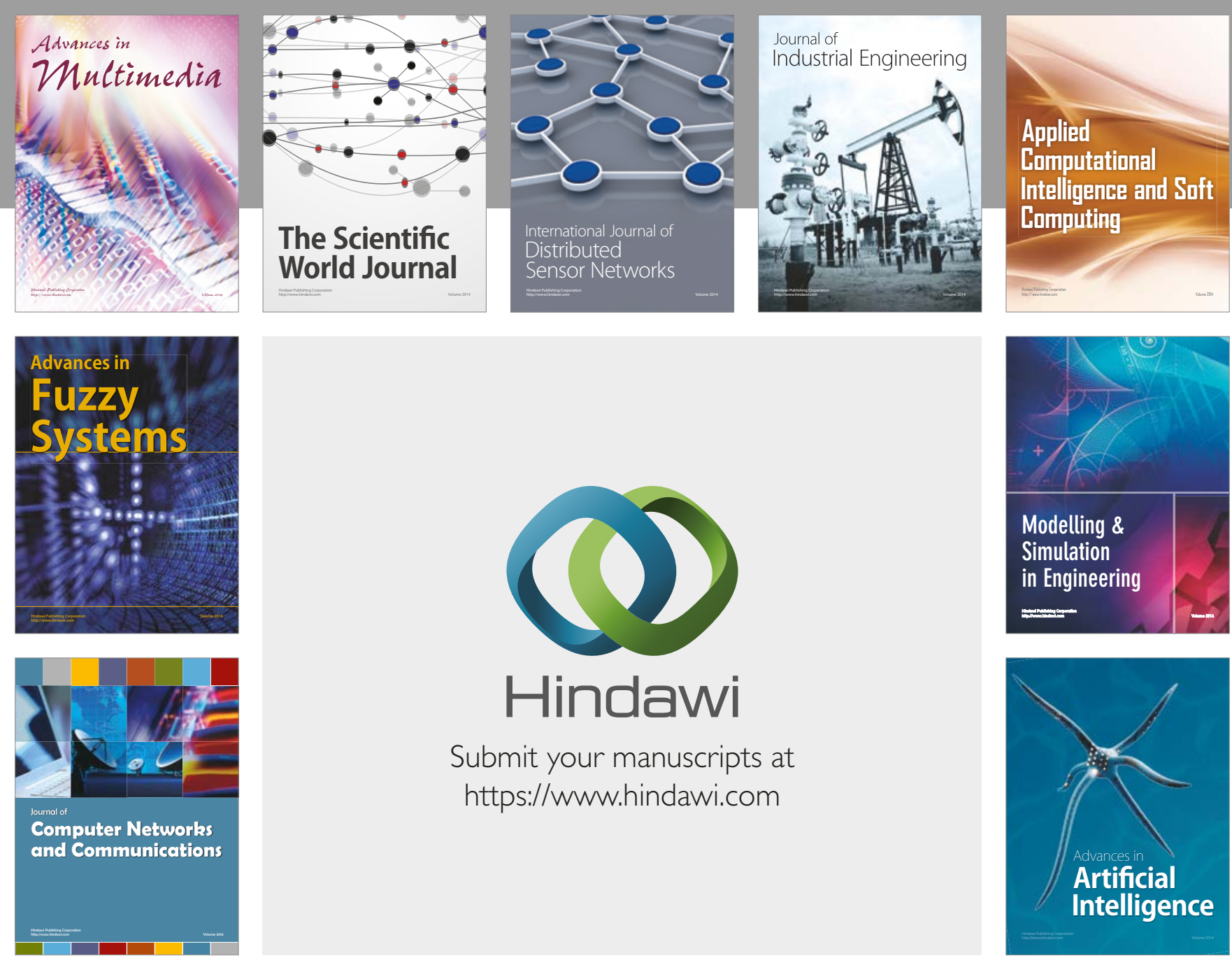

\section{Hindawi}

Submit your manuscripts at

https://www.hindawi.com
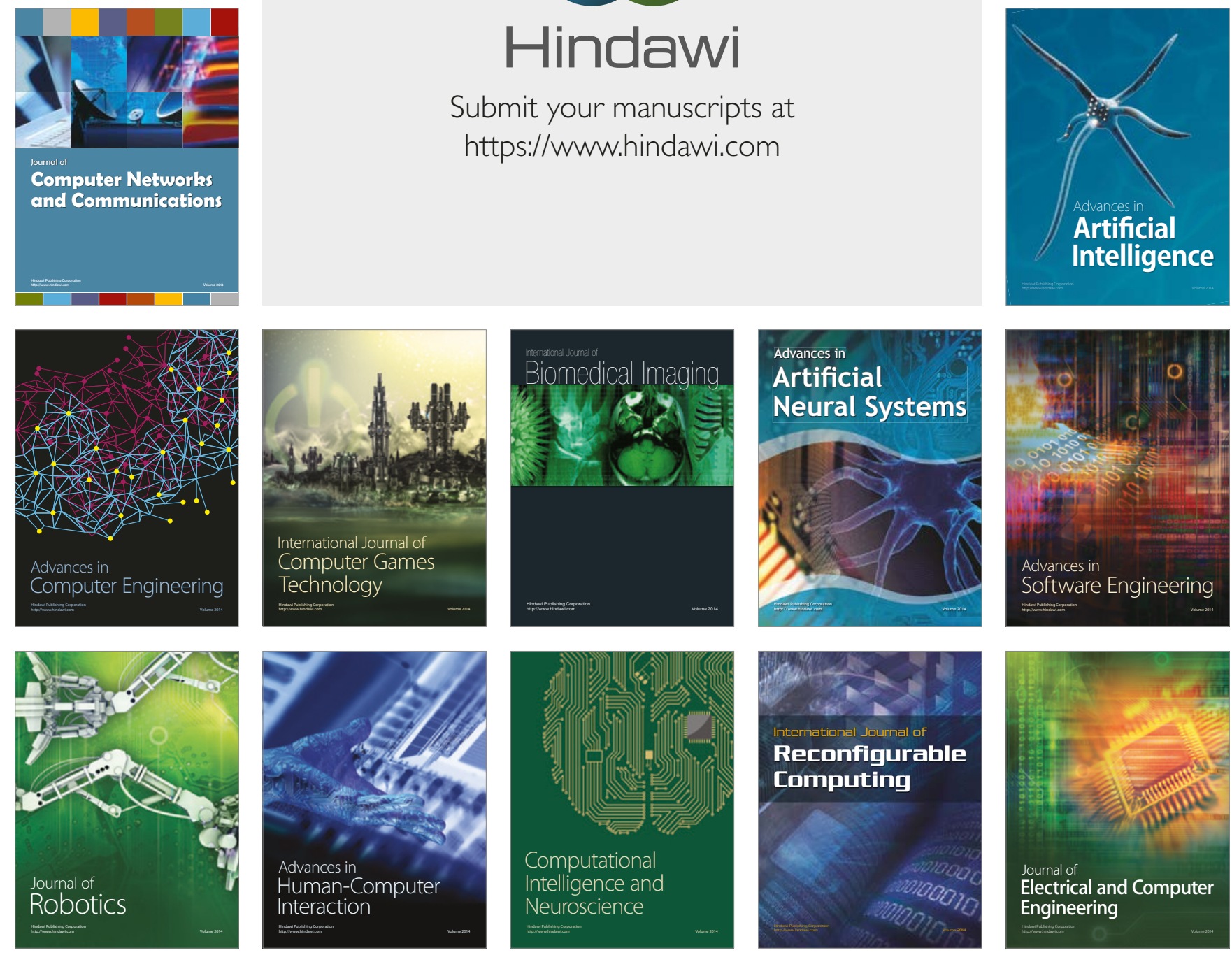